\title{
Cheaters shape the evolution of phenotypic heterogeneity in Bacillus subtilis biofilms
}

\author{
Marivic Martin $\mathbb{1}^{1,2} \cdot$ Anna Dragoš ${ }^{1} \cdot$ Simon B. Otto $\mathbb{C}^{1} \cdot$ Daniel Schäfer ${ }^{2} \cdot$ Susanne Brix $\mathbb{D}^{3} \cdot$ Gergely Maróti $^{4} \cdot$ \\ Ákos T. Kovács $\mathbb{B}^{1,2}$
}

Received: 13 February 2020 / Revised: 6 May 2020 / Accepted: 13 May 2020 / Published online: 1 June 2020

(c) The Author(s), under exclusive licence to International Society for Microbial Ecology 2020

\begin{abstract}
Biofilms are closely packed cells held and shielded by extracellular matrix composed of structural proteins and exopolysaccharides (EPS). As matrix components are costly to produce and shared within the population, EPS-deficient cells can act as cheaters by gaining benefits from the cooperative nature of EPS producers. Remarkably, genetically programmed EPS producers can also exhibit phenotypic heterogeneity at single-cell level. Previous studies have shown that spatial structure of biofilms limits the spread of cheaters, but the long-term influence of cheating on biofilm evolution is not well understood. Here, we examine the influence of EPS nonproducers on evolution of matrix production within the populations of EPS producers in a model biofilm-forming bacterium, Bacillus subtilis. We discovered that general adaptation to biofilm lifestyle leads to an increase in phenotypical heterogeneity of eps expression. However, prolonged exposure to EPS-deficient cheaters may result in different adaptive strategy, where eps expression increases uniformly within the population. We propose a molecular mechanism behind such adaptive strategy and demonstrate how it can benefit the EPS producers in the presence of cheaters. This study provides additional insights on how biofilms adapt and respond to stress caused by exploitation in long-term scenario.
\end{abstract}

\section{Introduction}

Cooperative interactions are prevalent for all life forms [1], even for simple microbes that often exist in communities of matrix bound surface-attached cells called biofilms [2-6]. However, when costly products such as siderophores [7, 8],

Supplementary information The online version of this article (https:// doi.org/10.1038/s41396-020-0685-4) contains supplementary material, which is available to authorized users.

\section{Ákos T. Kovács}

atkovacs@dtu.dk

1 Bacterial Interactions and Evolution Group, Department of Biotechnology and Biomedicine, Technical University of Denmark, 2800 Kongens Lyngby, Denmark

2 Terrestrial Biofilms Group, Friedrich Schiller University Jena, 07743 Jena, Germany

3 Disease Systems Immunology Group, Department of Biotechnology and Biomedicine, Technical University of Denmark, 2800 Kongens Lyngby, Denmark

4 Institute of Plant Biology, Biological Research Center of the Hungarian Academy of Sciences, Szeged 6726, Hungary extracellular polymeric substances $[9,10]$, digestive enzymes [11], and signaling molecules [12,13] are secreted and shared, cooperative behavior becomes susceptible to cheating [2,14-16], where mutants defective in cooperation can still benefit from cooperative community members $[4,5,17]$. It has been shown that spatially structured biofilms, where interactions with clone mates are common and diffusion of public goods is limited, may serve as natural defense against cheating [18-20]. However, long time scale studies have recently reported that biofilm defectors can spontaneously emerge and spread in biofilms by exploiting other matrix-proficient lineages [21-24]. In fact, a pioneering microbial evolution study on Pseudomonas fluorescens has already pointed toward dynamic evolutionary interplay between cooperation and exploitation in a biofilm mat [25], where emergence of cellulose overproducer (wrinkly) allowed mat formation, but also created an opportunity for exploitation by nonproducers (smooth), eventually leading to so-called "tragedy of the commons" $[4,26,27]$.

Taken together, biofilms are a suitable model to understand social interactions in an evolutionary time scale [23, 28-31]. Modeling and empirical data confirm that 
mutualism (beneficial to both actor and recipient) and altruism (beneficial to recipient but not to actor) play crucial roles in biofilm enhancement [32], but at the same time can lead to biofilm destabilization [25]. Can cooperators evolve tactics to evade exploitation and, in turn, can cheats utilize evolution to enhance their selfish actions?

Recent studies showed that in well-mixed environment, cooperators adapt to cheats by reducing cooperation $[14,15,33]$. Such reduction could be achieved by various strategies, for instance decrease in motility [15], downregulation or minimal production in public goods $[14,15,33]$, upregulation of other alternative public goods [14], or bi-stable expression in virulence gene [2]. Interestingly, populations of cooperators often exhibit phenotypic heterogeneity at the single-cell level [34, 35]. Therefore, an alternative and simple mechanism to modulate levels of cooperation in a population would be through changes in phenotypic heterogeneity pattern. However, the long-term effects of cheats on costly goods' expression at individual cell level have never been examined. Understanding how heterogeneity of gene expression within the population is affected in the presence of cheats would provide better insight on microbial adaptation and stress response mechanisms.

Here, we address this question using pellicle biofilm model of Bacillus subtilis (B. subtilis) [36, 37]. Pellicle formation in $B$. subtilis involves, among others, aerotaxisdriven motility and subsequent matrix production [38]. Aerotaxis is important for oxygen sensing to aid cells reach the surface, while matrix formation is significant to sustain cells to adhere to the surface and to each other. Exopolysaccharide (EPS) is a costly public good in B. subtilis biofilms [10, 18, 39] and is heterogeneously expressed during biofilm formation with $\sim 40 \%$ of cells exhibiting the ON state [39, 40]. We aimed to investigate the cheatdependent alteration related to phenotypic heterogeneity in eps expression by the producer.

We reveal that cheating mitigation by the EPS producers involves a shift in phenotypic heterogeneity toward stronger eps expression, which can be achieved by a loss-of-function mutation in a single regulatory gene. Our study uncovers an alternative anti-cheating mechanism based on changes in public goods' expression pattern and highlights meandering trajectories prior cooperation collapse.

\section{Materials and methods}

\section{Bacterial strains and culture conditions}

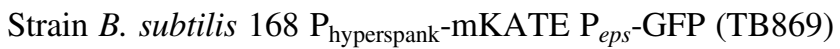
was obtained by transforming the laboratory strain, $B$. subtilis $168 \mathrm{P}_{\text {hyperspank-mKATE (TB49) }}$ [10, 18], with genomic DNA from NRS2243 (sacA:: $\mathrm{P}_{e p s A^{-}} g f p(\mathrm{Km}) h a g::$ cat) and selecting for $\mathrm{Km}$ resistance. The $\Delta r s i X$ strain with fluorescence reporters (TB959) was obtained by transforming TB869 with genomic DNA isolated from BKE23090 (168 trpC2 $\Delta r s i X:: e r m)$ [41]. Strains were maintained in LB medium (Lysogeny Broth (Lennox), Carl Roth, Germany), while $2 \times$ SG medium was used for biofilm induction [10]. The $\Delta$ eps strains (TB608) were created previously [10].

\section{Experimental evolution}

Eight biological replicates of the cocultures of 1:1 ratio of B. subtilis TB869 and TB608 were grown in 48-well plate containing $1 \mathrm{ml} 2 \times \mathrm{SG}$ medium at $30^{\circ} \mathrm{C}$ for 2 days. Pellicles were harvested into Eppendorf tubes containing $500 \mu \mathrm{l}$ sterile $2 \times$ SG medium and $100 \mu \mathrm{l}$ of sterile glass sand, vortexed for $90 \mathrm{~s}, 10 \mu \mathrm{l}$ fraction was transferred into $1 \mathrm{ml}$ $2 \times \mathrm{SG}$ medium of a 48 -well plate and incubated at $30^{\circ} \mathrm{C}$ static condition for 2 days. Such growth cycle was continuously repeated 35 times. As a control treatment, four biological replicates of mono-cultures of B. subtilis TB869 were evolved using the same transfer method. Every fifth transfer (five growth cycles), harvested cultures were mixed with $15 \%$ glycerol and stored at $-80^{\circ} \mathrm{C}$.

\section{Population ratio assay}

At every fifth transfer, pellicle biofilm productivities and relative frequencies of mutants and wild type (WT) were qualitatively assessed (colony forming units $(\mathrm{CFU}) / \mathrm{ml}$ ) using LB agar containing selective antibiotics. LB agar plates were incubated at $37^{\circ} \mathrm{C}$ for $16 \mathrm{~h}$ and colonies were counted. Three single clones of WT and of Aeps per population per time point were isolated from plates and stored at $-80{ }^{\circ} \mathrm{C}$ in the presence of $15 \%$ glycerol.

\section{Pellicle competition assay/fitness assay}

Competition assays were performed as previously described [10]. Specifically, strains of interest were premixed at 1:1 ratio based on their $\mathrm{OD}_{600}$ values and the mixture was inoculated into $2 \times \mathrm{SG}$ medium at $1 \%$. Cultures were grown for $48 \mathrm{~h}$ under static conditions at $30^{\circ} \mathrm{C}$ and their relative frequencies were accessed using CFU counts (and selective antibiotics).

\section{Stereomicroscopy to assess competition of WT and $\Delta$ rsiX against $\Delta$ eps}

Fluorescent images of pellicles were obtained with an Axio Zoom V16 stereomicroscope (Carl Zeiss, Jena, Germany) equipped with a Zeiss CL 9000 LED light source and an 
AxioCam MRm monochrome camera (Carl Zeiss) and HE eGFP (excitation at $470 / 40 \mathrm{~nm}$ and emission at 525/50 nm), and HE mRFP (excitation at $572 / 25 \mathrm{~nm}$ and emission at $629 / 62 \mathrm{~nm}$ ) filter sets. Images were taken at $3.5 x$ and $55 x$ magnifications. The exposure times for green and red fluorescence were set up to maximal possible values before reaching overexposure, using range indicator function. Zeiss software was used to obtain overlaid, artificially colored images of both fluorescence channels.

\section{Qualitative assessment of eps expression pattern via laser scanning confocal microscopy}

Single isolates of evolved WT (TB869) obtained from population ratio assay were allowed to form 1-day-old pellicle. Harvested pellicles were subjected to microscopic analysis using an Axio Observer 780 Laser Scanning Confocal Microscope (Carl Zeiss) equipped with a PlanApochromat $63 \times / 1.4$ Oil DIC M27 objective, an argon laser for stimulation of fluorescence (excitation at $488 \mathrm{~nm}$ for green fluorescence and $561 \mathrm{~nm}$ for red fluorescence, with emission at 528/26 and $630 / 32 \mathrm{~nm}$, respectively). Zen 2012 Software (Carl Zeiss) and FIJI Image J Software [42] were used for image recording and processing, respectively.

\section{Flow cytometry and data analysis}

Frozen stocks of evolved populations were transferred onto LB-agar plates containing kanamycin $(5 \mu \mathrm{g} / \mathrm{ml})$ to select solely for WT colonies. The plates were incubated overnight at $37^{\circ} \mathrm{C}$, followed by inoculation of ten randomly selected single colonies into $2 \times \mathrm{SG}$ medium. After $24 \mathrm{~h}-$ incubation at $30^{\circ} \mathrm{C}$, the pellicles were harvested, sonicated, and diluted accordingly. Flow cytometry was performed using BD FACSCanto II (BD Biosciences). To separate bacterial cells from noise, mKate-fluorescence (constitutively expressed reporter) and GFP fluorescence $\left(\mathrm{P}_{e p s^{-}}\right.$ GFP promoter fusion) were recorded, gating was setup for mKate-positive objects, and GFP signal was measured within these objects. Histograms of $\mathrm{P}_{e p s}$-GFP were created in OriginPro using the same binning intervals for all samples. To remove sample size differences (different amounts of measured objects), histograms were normalized to maximum count, described as Normalized Frequency.

To obtained an average distribution image of eps expression within populations, a mean count for each histogram bin was calculated (by averaging individual counts within this bin obtained for single isolates), resulting in mean distribution of single-cell level eps expression per population. Such "averaged" histograms were used solely for visual representation of data and not for statistical analysis.

\section{Genome re-sequencing and genome analysis}

Genomic DNA of single isolates from selected evolved populations were extracted using Bacterial and Yeast Genomic DNA kit (EURx) directly from $-80^{\circ} \mathrm{C}$ stocks grown in LB medium for $5 \mathrm{~h}$ at $37^{\circ} \mathrm{C}$ with shaking at 220 rpm. For population sequencing analysis, $\sim 100$ colonies belonging to the evolved populations were harvested into 2 $\mathrm{ml} \mathrm{LB}$ broth and incubated at $37{ }^{\circ} \mathrm{C}$ shaking at $220 \mathrm{rpm}$ for $2-3 \mathrm{~h}$. Re-sequencing was performed on an Illumina NextSeq instrument using V2 sequencing chemistry $(2 \times$ $150 \mathrm{nt})$. Base calling was carried out with "bcl2fastq" software (v.2.17.1.14, Illumina). Paired-end reads were further analyzed in CLC Genomics Workbench Tool 9.5.1. Reads were quality-trimmed using an error probability of 0.05 (Q13) as the threshold. Reads that displayed $\geq 80 \%$ similarity to the reference over $\geq 80 \%$ of their read lengths were used in mapping. Quality-based SNP and small In/Del variant calling were carried out requiring $\geq 10 \times$ read coverage with $\geq 25 \%$ variant frequency. Only variants supported by good quality bases $(\mathrm{Q} \geq 30)$ on both strands were considered. Gene functions (product names) in SI Appendix Datasets were reported based on SubtiWiki [43].

\section{Statistical analysis}

Statistical differences between two experimental timepoints of the same experimental groups (e.g., changes in relative frequencies of WT and $\Delta e p s$ during evolution) were accessed using pair-sample $t$-test. Statistical differences between two experimental groups were calculated using two-sample $t$-test. To compare multiple samples with WT, we used one-way repeated measures ANOVA and Dunnett test. ANOVA and Tukey test were used for multiple samples comparisons.

For analysis of $\mathrm{P}_{e p s}$-GFP expression in evolved populations, we used ten randomly picked single colonies, cultivated from the frozen stocks (tenth transfer) on LB-agar plate with appropriate selection marker (selecting against $\Delta e p s)$. All flow cytometry data of $\mathrm{P}_{e p s}$-GFP expression were transferred to histograms, and fitted to Gauss function. Differences in average eps expression per population compared with $\mathrm{WT}_{\mathrm{anc}}$ and differences in single-cell level distribution of eps expression compared with $\mathrm{WT}_{\text {anc }}$ were calculated using one-way repeated measures ANOVA and mean comparison by Dunnett test. Deviation from WT-like distribution was assessed from changes in adjusted Rsquared (Adj. R. Sq) values for Gauss fitting of flow cytometry data. All evolved populations, where average Adj. R. Sq was significantly lower compared with WT ancestor, were suspected to have evolved different phenotypic heterogeneity pattern of eps expression. Corresponding histograms were visually inspected, classified as 
potentially bimodal, and subjected to multiple peak fitting. In all such cases, fit quality was improved (Adj. R. Sq > 0.98). Mean expression for eps-low and eps-high subpopulations was compared by ANOVA, Tukey test. No statistical methods were used to predetermine sample size and the experiments were not randomized. One data point was removed from the $\mathrm{P}_{e p s}$-GFP flow cytometry dataset of $\Delta r s i X$ as a significant outlier $\left(P<4.5 \times 10^{-7}\right)$ confirmed by Grubbs test. All statistical tests and data fitting were performed using OriginPro 2018 software.

\section{Results}

\section{Cheaters modulate evolution of phenotypic heterogeneity of eps expression in the WT}

EPS is one of the major components of B. subtilis biofilm matrix and mutants deficient in EPS production ( $\Delta$ eps) are not able to form pellicle biofilms (Supplementary Fig. 1). In line with previous results $[10,18,39]$, we confirmed that the $\Delta$ eps can take advantage of EPS-producing WT and incorporate into the pellicle biofilm, resulting in lower productivity of the WT (Supplementary Fig. 1b) and reduced surface complexity of the pellicle (Supplementary Fig. 1a). Interestingly, despite pellicles formed by the WT $+\Delta$ eps lacked surface complexity and were more fragile compared with the WT monoculture pellicles (as easily observed during sampling), the total numbers of viable cells (our productivity measure) in the WT and mixed pellicles were similar (Supplementary Fig. 1b). This indicates high carrying capacity of the WT to support surface colonization by $\Delta$ eps.

We were further interested if such social cheating could leave a phenotypic or genetic fingerprint in the population of the WT B. subtilis. Previous studies have shown that cooperators can adapt to presence of cheats, for example, by decreasing the amount of released public goods and therefore minimizing cheating opportunities [2, 14, 15]. As $B$. subtilis exhibits phenotypic heterogeneity in eps matrix gene expression [39, 40] (Fig. 1a), we investigated how such heterogeneous expression is influenced by the presence of cheats in an evolutionary perspective.

To address this question, we cocultured the EPS producers (WT) and cheaters ( $\Delta e p s)$ for ten biofilm growth cycles ( 60 generations) starting at 1:1 ratio (see "Methods"). Based on previous studies [21, 44], we assumed that this

A)

B)

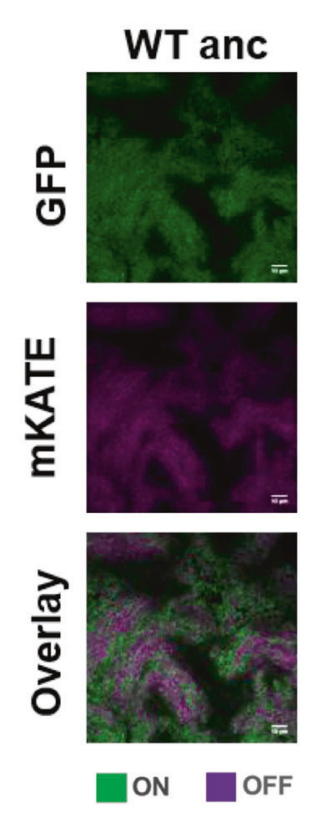

Fig. 1 Pellicle productivity and phenotypic heterogeneity. a Total colony forming unit per $\mathrm{ml}$ of WT and $\Delta$ eps in 48-h-old pellicles nonevolved (start) $(n=9)$, after experimental evolution at fifth $(n=8$ populations), and tenth transfer ( $n=7$ populations). One population after tenth transfer was unable to form pellicle attributed to WT being totally outnumbered by $\Delta$ eps. Dots represent data obtained for all individual populations, while columns represent averages. Error bars correspond to standard error. Levels of $\Delta e p s$ at the start, fifth, and tenth transfers were $82 \%, 76 \%(p<0.29$ compared with start, pairsample $t$-test), and $83 \%(p<0.42$ compared with start, pair-sample $t$ test). b Pellicles formed by $\mathrm{WT}^{\mathrm{mKATE}} \mathrm{P}_{e p s}$-GFP viewed under the confocal laser scanning microscope. Cells constitutively expressing mKATE are represented in magenta (OFF cells) and eps-expressing cells (ON cells) are represented in green. Scale bar $10 \mu \mathrm{m}$. (Color figure online). 
evolutionary timeframe will be sufficient for evolution of adaptive mechanisms in the WT in response to social cheating, at the same time preventing the diversification of the WT into a biofilm-deficient morphotype, which can be observed on longer evolutionary timescales [21].

During ten-cycle cocultivation of WT and $\Delta e p s$ strain, we observed a general trend of declining pellicle productivity (Fig. 1b). The relative frequency of EPS nonproducers in biofilms was maintained at the high level across all parallel populations $(82 \%$ at the start, $76 \%$ after fifth transfer with $p<$ 0.29 , and $83 \%$ after tenth transfer with $p<0.42$ ) (Fig. 1b), indicating that the WT strain was constantly exposed to social cheating throughout the experiment.

Using confocal laser scanning microscopy, qualitative assessment of randomly selected isolates revealed that early populations of the EPS producers (5-10 transfers) exhibited different phenotypes compared with the WT ancestral strain $\left(\mathrm{WT}_{\mathrm{anc}}\right.$ ) (Supplementary Fig. 2). To obtain a quantitative comparison of single-cell level expression of eps in the $\mathrm{WT}_{\text {anc }}$ vs. evolved WT populations, we performed flow cytometry measurements of $\mathrm{P}_{e p s}$-GFP harboring strains in pellicles formed by ten randomly selected isolates per population (Supplementary Fig. 3).

First, we noticed that in most strains isolated from the control evolved populations exhibited an increase in phenotypic heterogeneity in eps expression as compared with the WT ancestor (Fig. 2a, b; Supplementary Fig. 3, Supplementary Dataset 1). Increased level of phenotypic heterogeneity was evident from frequency distributions of single-cell $\mathrm{P}_{e p s}$-GFP expression data (Fig. 2b). Specifically, while single-cell level distribution of $\mathrm{P}_{e p s}$-GFP in the WT ancestor, ideally fitted the Gauss function (Adj. R. Sq = $0.99 \pm 0.01$ ), indicating presence of a single population of eps-expressing cells (Fig. 2a, b; Supplementary Fig. 3). This was no more the case for the populations of WT evolved without cheater (C1, C2, and C4; Adj. R. Sq: 0.88-0.93), where distributions of $\mathrm{P}_{e p s}$-GFP shifted away from the unimodal distribution (as could be quantitatively expressed in significantly lower Adj. R. Sq values, therefore lower percentage of data points fitting into Gauss model) toward bimodal distributions (Fig. 2a, b; Supplementary Fig. 3; Supplementary Dataset 1). Presence of two frequency peaks, one at low and second at high $\mathrm{P}_{e p s}$-GFP fluorescence levels, was particularly evident in some of the control populations (Supplementary Fig. 3). Similar change was noticed for two WT populations evolved with cheaters (Fig. 2a; Supplementary Fig. 3, Pop1 and Pop8; Adj. R. Sq: 0.87-0.94). In the aforementioned populations, the eps expression was also rather bimodal, distributed between low-eps and high-eps subpopulations (Fig. 2a, b; Supplementary Fig. 3; Supplementary Dataset 1). Importantly, these bimodal populations evolved alone $(\mathrm{C} 1, \mathrm{C} 2$, and $\mathrm{C} 4)$ or co-evolved with $\Delta$ eps (Pop1 and Pop8), were similar in terms of eps expression levels or ratios of low-eps and higheps subpopulations (Supplementary Dataset 1). In addition, an average within-population eps expression increased in four out of seven populations that evolved with cheater, but only in one control population evolved without cheats (Fig. 2c, Supplementary Dataset 1). Finally, higher levels of eps expression positively correlated with lower levels of withinpopulation heterogeneity (therefore higher Adj. R. Sq values) (Pearson correlation coefficient $0.33 ; p<0.0004$ ).

Altogether, most populations co-evolved with cheater showed a significant increase in eps expression levels, retaining WT-like phenotypic heterogeneity pattern (no significant changes in Adj. R. Sq for unimodal Gauss distribution fitting); we define this evolved phenotype as high eps. On the contrary, in majority of control populations (evolved without cheaters) phenotypic heterogeneity level increased, without significant increase in mean eps expression (Fig. 2; Supplementary Fig. 3; Supplementary Dataset 1); we define this evolved phenotype as heterogenous eps.

\section{Mutations in rsiX lead to high-eps phenotype}

To unravel the genetic basis of the high-eps phenotype that evolved in presence of cheats, several single isolates from the evolved populations were subjected to genome resequencing (for details see "Methods"). The comparative analysis of sequencing data revealed that Population 3 and Population 7, co-evolved with $\Delta e p s$, shared mutations in $r s i X$ gene (Supplementary Dataset 2). The rsiX gene encodes for an anti-sigma factor that controls the activity of extracellular cytoplasmic function (ECF) sigma factor $\mathrm{X}$ which is involved in cationic antimicrobial peptide resistance important for cell envelope stress response [45]. Detected mutations resulted either in substitution of Valine 106 to Alanine or frameshift mutations in Serine 104 or Isoleucine 347 that could lead to change or loss of anti-SigX function. Indeed, we were able to recreate the evolved higheps phenotype in the pellicle solely by deleting the rsiX gene in the WT ancestor (Fig. 3a, b). Interestingly, a different type of frameshift mutation in Lysine 200 was found in one population of evolved WT alone but this population demonstrated a bimodal phenotypic heterogeneity pattern (Fig. 2; Supplementary Fig. 3; Supplementary Dataset 1), suggesting that only certain types of mutations in $r s i X$ lead to the uniform shift in eps expression or additional mutations have antagonistic effects in this isolate.

\section{Mutation in rsiX contributes to competitive advantage of producer strains against cheats}

As mutation in $r s i X$ resulted in high-eps phenotype that may be linked to elevated secretion of EPS, we hypothesized that $\Delta r s i X$ producers could support the spread of cheats. To 
A)

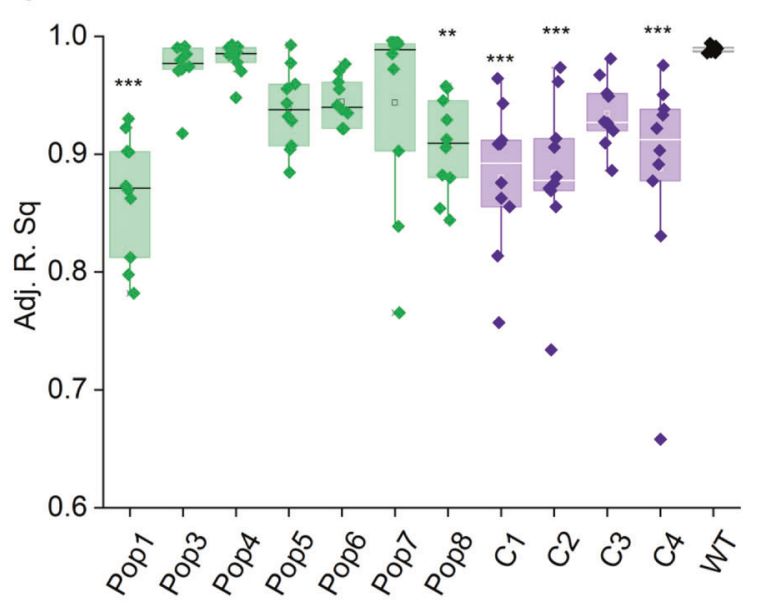

C)

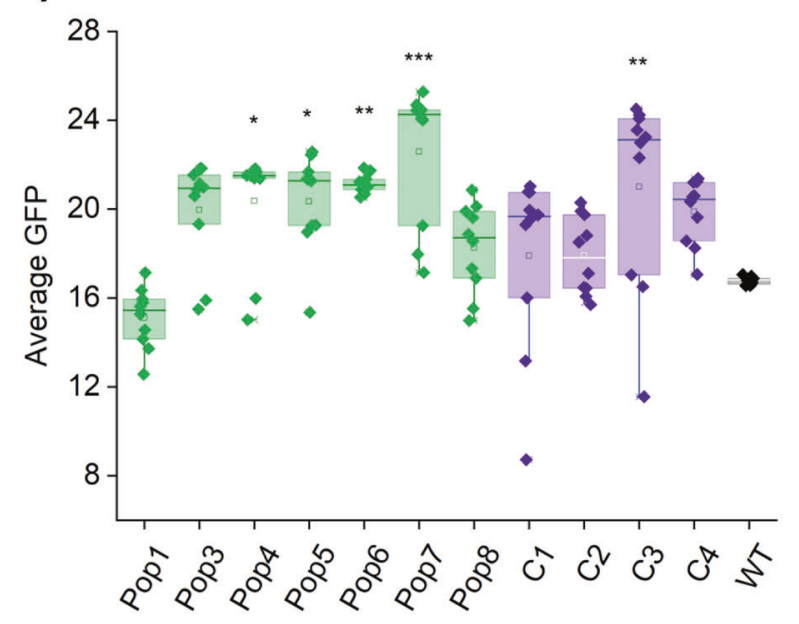

B)
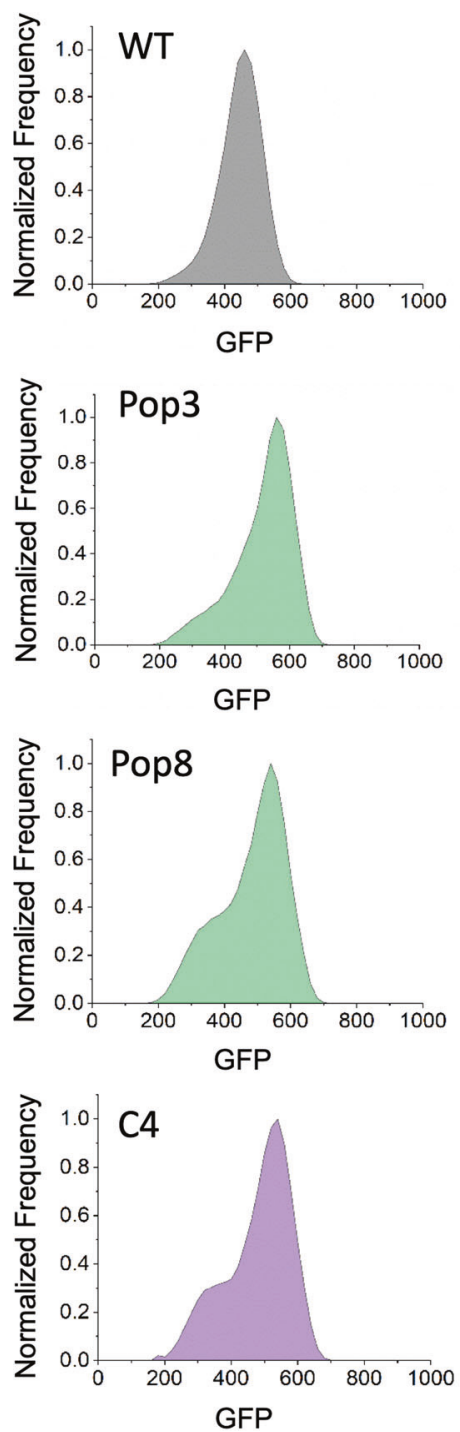

Fig. 2 Evolutionary changes in phenotypic heterogeneity pattern and expression of eps. a Changes in distribution of $\mathrm{P}_{e p s}$-GFP signal in co-evolved (Pop1-Pop8) and evolved $(\mathrm{C} 1-\mathrm{C} 4)$ WT populations compared with the $\mathrm{WT}_{\text {anc }}$ manifested as a decline of adjusted R-square values for Gauss model fitting. b Flow cytometry analysis showing average distributions of $\mathrm{P}_{e p s}-\mathrm{GFP}$ in $\mathrm{WT}_{\text {anc }}$ (dark gray), WT evolved with cheaters, Pop3 and Pop8 (green) and WT evolved without

better understand how ancestor WT and $\Delta r s i X$ interact with $\Delta e p s$, we cultivated the $\Delta e p s$ in presence of EPS-containing supernatants obtained from the WT and $\Delta r s i X$ (Supplementary Fig. 4). Both supernatants could partially restore pellicle formation by $\Delta e p s$ resulting in similar productivities of $\Delta e p s$, thereby not supporting our hypothesis on improved performance of the mutant in presence of higheps $\Delta r s i X$ strain.

In order to determine the effect of $r s i X$ deletion on fitness of the WT in presence of cheats, we performed a series of competition assays. Apparently, the $\Delta r s i X$ showed two-fold cheaters, C4 (purple). c Average $\mathrm{P}_{e p s}$-GFP expression levels in coevolved (Pop1-Pop8) and evolved (C1-C4) WT populations compared with the $\mathrm{WT}_{\mathrm{anc}}$, calculated from mean values of $\mathrm{P}_{e p s}$-GFP expression within ten single isolates. For a and $\mathbf{c}$ panels $* p<0.05$; $* * p<0.01 ; * * * p<0.001$ compared with the $\mathrm{WT}_{\text {anc }}$ (one-way repeated measures ANOVA, Dunnett test). All data and corresponding $p$ values are provided in Supplementary Data 1. (Color figure online).

increase in relative frequency (40\%) (Fig. 4a; Supplementary Fig. 5) when competed against the $\Delta e p s$, as compared with the WT ancestor (20\%). In addition, even higher fitness improvement was observed for the WT co-evolved with cheats fifth transfer and tenth transfer, mutually with occurrence of high-eps phenotype in those populations. This was not the case for the WT evolved alone at fifth transfer (20\%) (Fig. 4a; Supplementary Fig. 5). These results suggest that $r s i X$ mutation, which is associated with high-eps phenotype, does not fully explain, but contributes to the early improvement of WT competitive strategies against cheats. 
A)
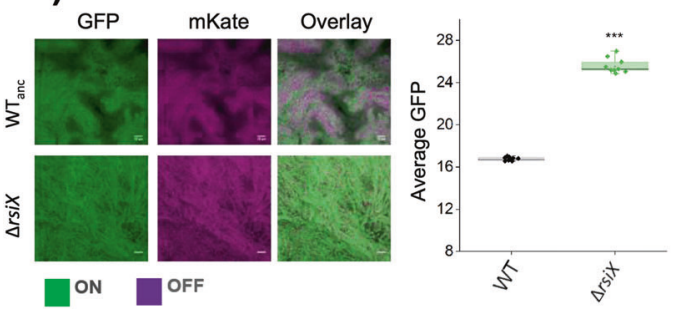

B)

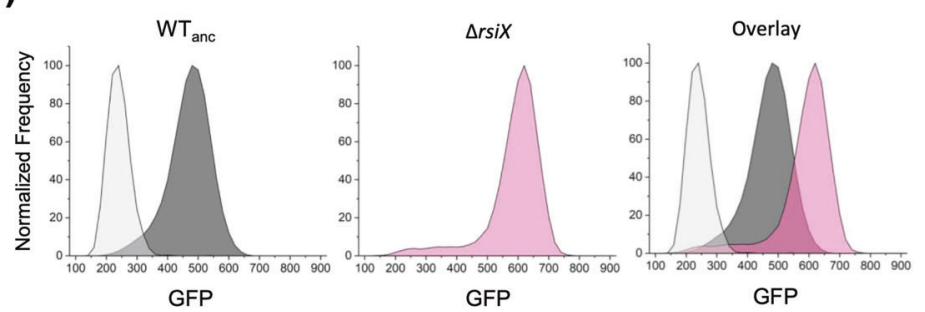

Fig. 3 Effect of rsiX deletion on eps expression in pellicles. a Qualitative assessment of eps gene expression based on confocal laser scanning microscopy of pellicles formed by $\Delta r s i X$ showing high eps compared with $\mathrm{WT}_{\text {anc }}$. Cells constitutively expressing mKATE (OFF) are shown in magenta and eps-expressing cells (ON) are represented in green. Scale bar $10 \mu \mathrm{m}$. Right panel: average $\mathrm{P}_{e p s}$-GFP expression levels in $\mathrm{WT}_{\mathrm{anc}}$ and $\Delta r s i X$ the $\mathrm{WT}_{\text {anc }}$ calculated from mean values of $\mathrm{P}_{e p s}-\mathrm{GFP}$ expression within ten single isolates. $* * * p<0.001$ (two-sample $t$-test). All data and corresponding $p$ values are provided in Supplementary Data 1 . b Flow cytometry results showing average distribution of fluorescence intensities of $\mathrm{WT}_{\mathrm{anc}}$ cells (dark gray), $\Delta r s i X$ cells (pink), and overlay of the two in comparison to WT nonlabeled (light gray). (Color figure online).
A)

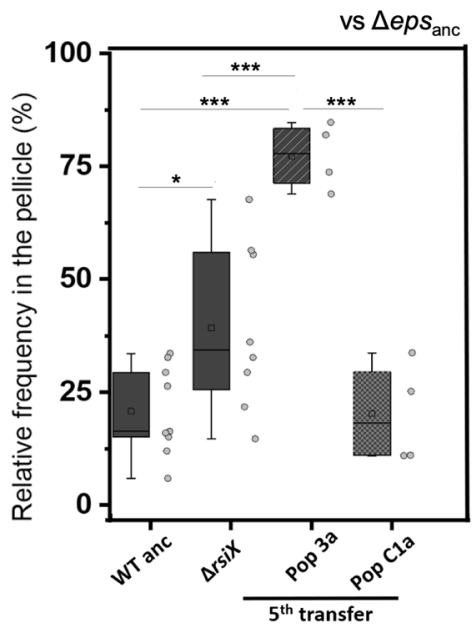

Fig. 4 Performance of evolved WT and $\Delta r s i X$ in cocultures with $\Delta$ eps $_{\text {anc. }}$ a Relative frequencies of single isolates belonging to producer populations $\left(\mathrm{WT}_{\text {anc }}(n=9), \Delta r s i X(n=8)\right.$, WT evolved with (Pop3a; $n=4)$ and without cheaters (PopC1a; $n=4)$ ) in mixed pellicles with $\Delta e p s_{\text {anc. }}$ b Productivity assay based on total $\mathrm{CFU} / \mathrm{ml}$ of pellicles of cocultures of $\Delta e p s_{\text {anc }}$ and single isolates belonging to producer populations $\left(\mathrm{WT}_{\text {anc }}(n=9), \Delta r s i X(n=8)\right.$, WT evolved with

It is worth to mention that we could not detect any significant fitness costs or benefits linked to rsiX deletion in pairwise competition between $\Delta r s i X$ and WT in the liquid medium (Supplementary Fig. 6; relative fitness of $\Delta r s i X=$ $1.00 \pm 0.02 \mathrm{SD}$ ). Furthermore, we did not observe significant differences in productivities of WT and the $\Delta r s i X$ mutant, when grown in monoculture pellicles (Fig. 4b), indicating that positive effect of rsiX mutation only manifests in presence of cheats. Similarly, different relative frequencies of $\Delta$ eps in pellicles formed by the ancestor or evolved matrix producers, did not result in different productivities of mixed pellicles (Supplementary Fig. 7). These results suggest that high-eps phenotypes are vested on the
B)

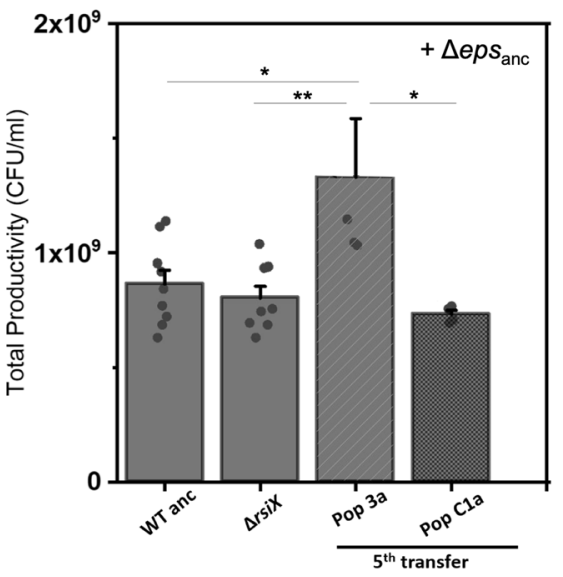

(Pop3a; $n=4)$ and without cheaters (PopC1a; $n=4)$ ). Mean is represented in square within the box plots; median is denoted by horizontal line inside the boxes; whiskers represent the min and max; error bars in bar graph are based on standard error; single dots represent the individual data points. For $\mathbf{a}$ and $\mathbf{b}$ panels: $* p<0.05$; $* * p<0.01 ; * * * p<0.001$ (ANOVA, Tukey test).

increase in eps-expressing cells or limiting the spread of cheats but do not result in an increase in total yield.

It was previously demonstrated that increased matrix production can allow favorable positioning of a bacterial strain in the biofilm, thereby providing fitness advantage [46]. To test whether high-eps phenotype can allow better positioning of the $\Delta r s i X$ in presence of $\Delta e p s$, we visualized $48 \mathrm{~h}$ grown pellicles formed by $\Delta r s i X: \Delta e p s$ and WT: $\Delta e p s$ mixtures inoculated at 1:1 initial frequencies (Fig. 5; Supplementary Fig. 8). While WT and $\Delta$ eps were "well-mixed" with both strains present on the oxygen-rich surface of the pellicle, the $\Delta r s i X$ strain clearly dominated over the $\Delta e p s$ occupying majority of the biofilm surface and marginalizing 
Fig. 5 Effect of $r s i X$ deletion on positioning of EPS producers in the pellicle. Competition assay between $\mathrm{WT}+\Delta$ eps and $\Delta r s i X+\Delta e p s$. Strains labeled with constitutively expressed GFP and mKate proteins were inoculated in $1: 1$ initial frequency, pellicles were cultivated for $48 \mathrm{~h}$ at $30^{\circ} \mathrm{C}$ and visualized using

stereomicroscope. Upper panels represent controls (two isogenic WT or $\Delta r s i X$ strains labeled with different fluorescent markers), middle panel represents pellicles formed by $\mathrm{WT}+\Delta$ eps, and bottom panels represent pellicles formed by $\Delta r s i X+\Delta e p s$, each in two alternative combinations of fluorescent markers. Scale bar corresponds to $500 \mu \mathrm{m}$.

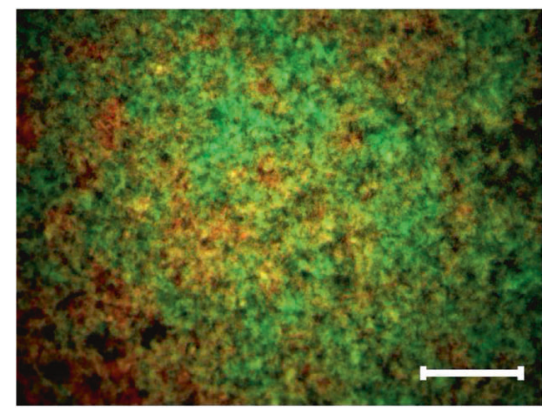

WT mKATE vs WT GFP

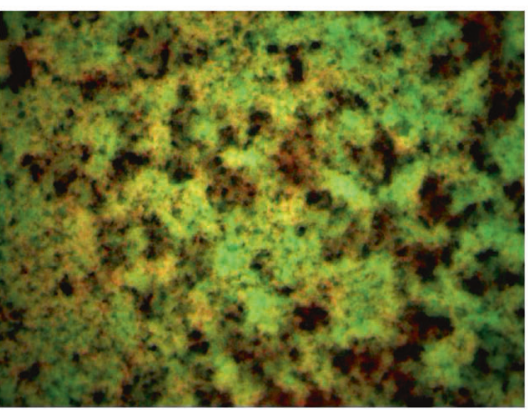

WT mKATE vs $\triangle$ eps GFP

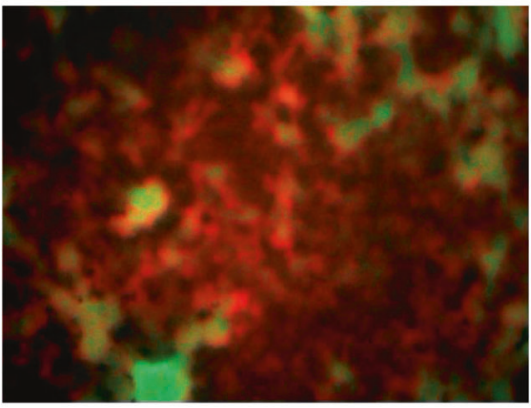

$\Delta r s i X$ mKATE vs $\triangle$ eps GFP

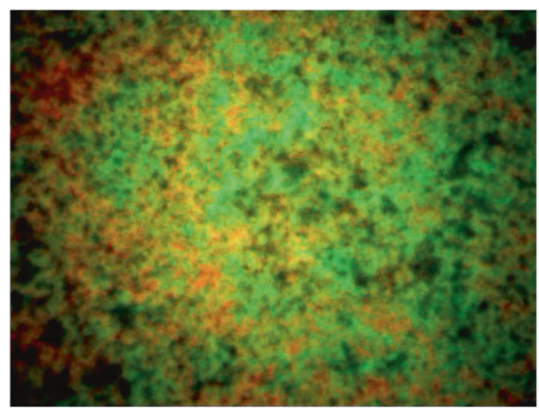

$\Delta r s i X$ mKATE vs $\Delta r s i X$ GFP

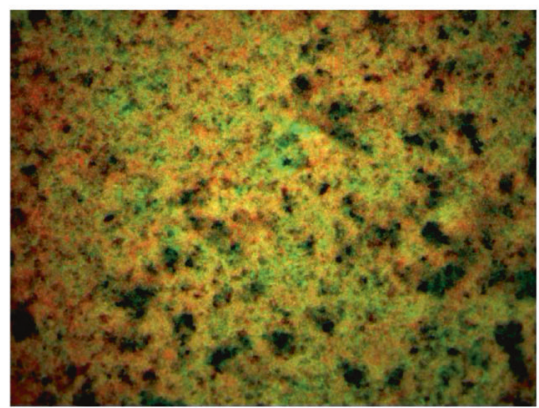

WT GFP vs $\triangle e p s$ mKATE

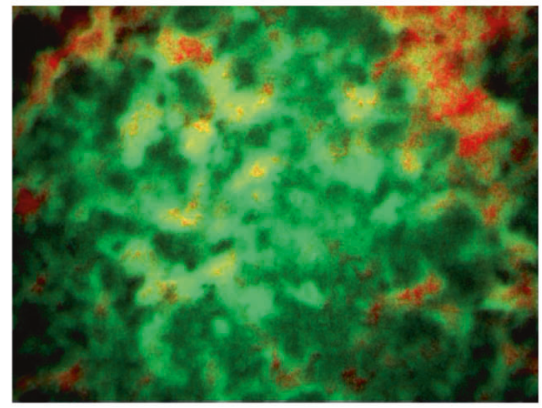

$\Delta r s i X$ GFP vs $\Delta$ eps mKATE the $\Delta$ eps into small clusters (Fig. 5; Supplementary Fig. 8). Therefore, deletion of rsiX and an associated high-eps phenotype provides fitness advantage in the presence of $\Delta e p s$ most likely by allowing the EPS producers to occupy upper, oxygen-rich layers of the pellicle. Therefore, $r s i X$ frameshift mutation found in certain co-evolved WT populations could be an adaptive mechanism to resist cheating by EPS-deficient strain.

\section{High-eps phenotype serves as a transient adaptive response to cheating}

As the evolved WT isolates carrying point mutation in $r s i X$ as well as the recreated $\Delta r s i X$ mutant (in ancestral genetic background) performed better in competition with $\Delta e p s$ as compared with the $\mathrm{WT}_{\mathrm{anc}}$, we reasoned that loss-of-function mutation in rsiX together with an associated high-eps phenotype might be an efficient evolutionary strategy against social cheating. Surprisingly, however, prolonged evolution experiment eventually led to so-called "tragedy of the commons" as the $\Delta$ eps mutant took over in six out of eight populations, completely abolishing the pellicle formation (Fig. 6; Supplementary Fig. 9).

To investigate the genetics behind this phenomenon all evolved WT populations from the last evolutionary time point (or the last time point prior the collapse) were resequenced. Curiously, in contrast to the WT populations that were outcompeted by $\Delta e p s$, both WT populations which resisted the invasion (Pop5 and Pop8), carried mutations in $y v r G$ gene (Supplementary Fig. 9; Supplementary Dataset 2) encoding for two-component histidine kinase involved in cell wall process. Finally, the rsiX mutation was not detected neither in the last populations before the collapse, with an exemption of population 7 , nor at the last transfer point for the non-collapse populations (Supplementary Dataset 2), implying that this mutation was lost in the late populations. 


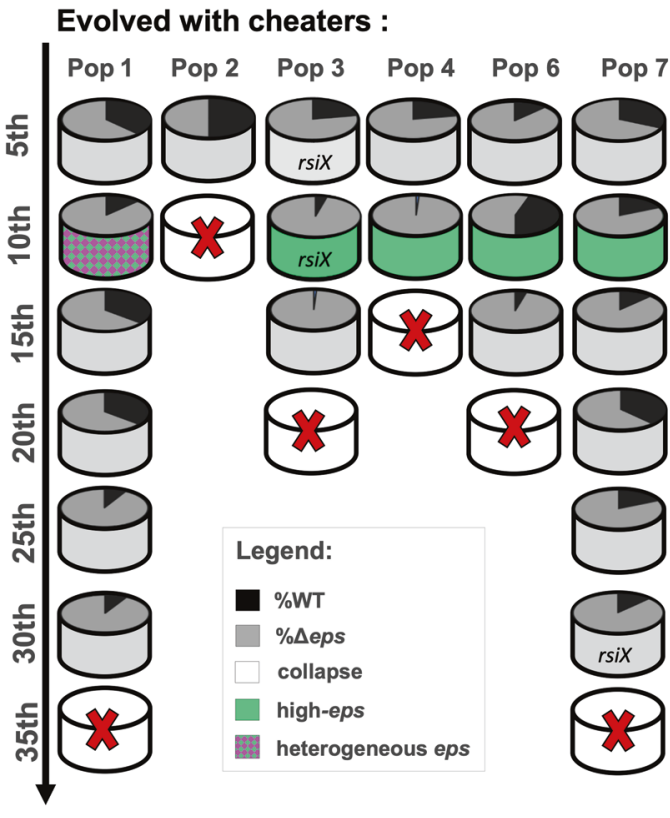

Fig. 6 Changes in relative frequencies and eps expression pattern during evolution with and without cheaters. Summary figure shows the population dynamics based on producer and cheater frequency per population from 5 th transfer to 35 th transfers for populations evolved with cheaters with collapse (Pop1-4, 6, and 7) and without collapse

\section{Discussion}

Studies on evolution of cooperative behavior are important to understand how social behaviors are shaped in longer time scale. Moreover, exploring long-term consequences of exposure to cheating allows to better understand how cooperation prevails in nature where environmental stress and exploitation exist inherently. Here, we took a reductionist approach, focusing on evolution of a single cooperative trait-the expression of eps, which plays a crucial role in biofilm lifestyle of $B$. subtilis and other bacteria. As we focused on the single-cell level expression of eps in multiple single strain, isolated from the ancestral or evolved populations, we could obtain a multi-level insight into evolutionary changes in eps expression. Our study revealed previously observed population-level diversification of matrix genes expression, indicating the strain-independence and reproducibility of adaptation in biofilms [21, 24]. Strikingly, next to population-level diversification, we also observe an increase in phenotypic heterogeneity of eps expression within single isolates. Based on coculture studies performed for WT and seps (this work), as well as for WT and spontaneously evolved biofilm-deficient lineage [21], we believe that low-eps subpopulations may be acting as conditional cheater, supported by "hyper-cooperative" subpopulations of high eps. The observation that isolates with the most pronounced phenotypic diversification

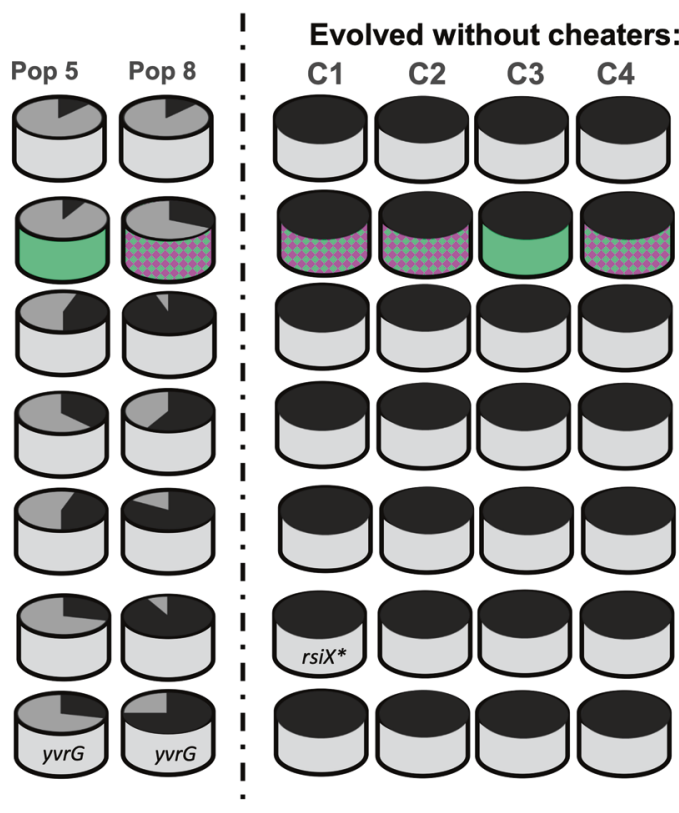

(Pop5 and 8) and populations evolved without cheaters (C1-C4) with indications of phenotypes based on high-eps or heterogenous-eps expression. Key mutations on single clone level of evolved WT and evolved $\Delta e p s$ or population level are specified. The ${ }^{*} r s i X$ mutation differs from mutation observed in strains evolved with cheaters.

pattern tend to show lower average eps expression, clearly depicts the latter as a consequence of diversification, and specifically emergence of low eps. It remains to be determined whether increased/reduced levels of eps expression translate into higher/lower amount of released EPS, but based on previous studies it is likely to be the case [44].

Previous evolution studies on cheater-cooperator interactions in spatially structured environment showed cheater mitigation via minimization of the cooperative trait $[2,14,15]$. On the contrary, here we show that cooperators respond to cheating by intensifying the cooperative behaviors through uniform shift toward higher eps expression. Further molecular analysis of the high-eps isolates strongly suggests that this phenotype is triggered by loss-of-function mutation in $r s i X$ gene. The product of $r s i X$ represses the activity of ECF sigma factor, SigX that is involved in cell envelope stress response against cationic antimicrobial peptides [47]. Importantly, SigX has been previously shown to induce expression of epsA-O in B. subtilis via a complex regulatory pathway involving Abh and SlrR [48], explaining the observed enhanced in eps gene expression in $r s i X$ mutant. Another example of matrix overproduction via ECF adaptation was also reported in gram-negative bacterium Pseudomonas aeruginosa ( $P$. aeruginosa) where mutations in another ECF called $\mathrm{Alg} \mathrm{T}$ led to alginate overproduction and increased resistance to antimicrobials [49]. Therefore, adaptive boosts in matrix production through modulation of ECF are not 
exclusive for B. subtilis, but seem to occur also in medically relevant gram-negative pathogens like $P$. aeruginosa.

In contrast to previous studies that addressed long-term cheating on diffusible siderophores [50-53], we explored evolutionary interplay between biofilm producers and nonproducers in structured environment. Our results support previous observations on evolution of specific cheatingresisting mechanisms in cooperators, pointing toward ubiquity of this phenomenon. In addition, our work brings up three major findings: (1) matrix producers can adapt to matrix nonproducers by shifting phenotypic heterogeneity toward increased levels of matrix expression, (2) high-eps phenotype is associated with favorable positioning of the matrix producers in the biofilm in presence of cheats, thereby limiting their numbers, (3) high-eps anti-cheating strategy is a short-term solution followed by tragedy of the commons. As EPS-deficient strain took over in all but two mixed populations (including populations, without $r s i X$ mutation and homogenous shift toward higher eps expression), we do not interpret the collapse as a direct consequence of mutation in $r s i X$ gene. However, we argue that an emergence of several matrix overproducing lineages, may facilitate the spread of cheats [21], especially if a substantial number of cells within the high-eps lineage serves as facultative (phenotypic) cheaters. As recently demonstrated, EPS deficiency is not a dead-end strategy for B. subtilis population, because alternative EPS-independent biofilm formation strategies can emerge by single amino acid change is TasA [44]. It remains to be discovered whether shifts in phenotypic heterogeneity in response to long-term cheating is general phenomenon that applies to different types of public goods.

Acknowledgements The authors thank James Gurney and other anonymous reviewers for their suggestions. This work was funded by the Deutsche Forschungsgemeinschaft (DFG) to ÁTK (KO4741/2.1) within the Priority Program SPP1617. MM was supported by a FEMS Research and Training Grant (FEMS-RG-2017-0054). This project has received funding from the European Union's Horizon 2020 research and innovation program under the Marie Skłodowska-Curie grant agreement No. 713683 (H.C. Ørsted COFUND to AD). Work in the laboratory of ÁTK is partly supported by the Danish National Research Foundation (DNRF137) for the Center for Microbial Secondary Metabolites.

Author contributions ÁTK conceived the project. MM, AD, SB, and DS performed the experiments. GM contributed with methods. MM, $\mathrm{AD}$, and ÁTK wrote the paper, with all authors contributing to the final version.

\section{Compliance with ethical standards}

Conflict of interest The authors declare that they have no conflict of interest.

Publisher's note Springer Nature remains neutral with regard to jurisdictional claims in published maps and institutional affiliations.

\section{References}

1. Bourke A. Principals of social evolution: Oxford series in ecology and evolution. Oxford: Oxford University Press; 2011.

2. Diard M, Garcia V, Maier L, Remus-Emsermann MNP, Regoes RR, Ackermann M, et al. Stabilization of cooperative virulence by the expression of an avirulent phenotype. Nature. 2013;494:353-6.

3. Nadell CD, Xavier JB, Foster KR. The sociobiology of biofilms. FEMS Microbiol Rev. 2009;33:206-24.

4. West SA, Diggle SP, Buckling A, Gardner A, Griffin AS. The social lives of microbes. Annu Rev Ecol Evol Syst. 2007;38:53-77.

5. West Sa, Griffin AS, Gardner A. Evolutionary explanations for cooperation. Curr Biol. 2007; 17:661-72.

6. Xavier J, Foster KR. Cooperation and conflict in microbial biofilms. Proc Natl Adac Sci USA. 2007;104:876-81.

7. Luján AM, Gómez P, Buckling A. Siderophore cooperation of the bacterium Pseudomonas fluorescens in soil. Biol Lett. 2015;11:20140934.

8. Weigert M, Kümmerli R. The physical boundaries of public goods cooperation between surface-attached bacterial cells. Proc R Soc B Biol Sci. 2017;284:20170631.

9. Dragoš A, Kovács ÁT. The peculiar functions of the bacterial extracellular matrix. Trends Microbiol. 2017;25:257-66.

10. Martin M, Dragoš A, Hölscher T, Maróti G, Bálint B, Westermann $\mathrm{M}$, et al. De novo evolved interference competition promotes the spread of biofilm defectors. Nat Commun. 2017;8:15127.

11. Folse HJ, Allison SD. Cooperation, competition, and coalitions in enzyme-producing microbes: social evolution and nutrient depolymerization rates. Front Microbiol. 2012;3:338.

12. Popat R, Crusz SA, Messina M, Williams P, West SA, Diggle SP. Quorum-sensing and cheating in bacterial biofilms. Proc R Soc B Biol Sci. 2012;279:4765-71.

13. Dogsa I, Oslizlo A, Stefanic P, Mandic-mulec I. Social interactions and biofilm formation in Bacillus subtilis. Food Technol Biotechnol. 2014;52:149-57.

14. O'Brien S, Luján AM, Paterson S, Cant MA, Buckling A. Adaptation to public goods cheats in Pseudomonas aeruginosa. Proc R Soc B Biol Sci. 2017;284:20171089.

15. Kümmerli R, Santorelli LA, Granato ET, Dumas Z, Dobay A, Griffin AS, et al. Co-evolutionary dynamics between public good producers and cheats in the bacterium Pseudomonas aeruginosa. J Evol Biol. 2015;28:2264-74.

16. Harrison F. Dynamic social behaviour in a bacterium: Pseudomonas aeruginosa partially compensates for siderophore loss to cheats. J Evol Biol. 2013;26:1370-8.

17. Hamilton WD. The genetical evolution of social behaviour. I. J Theor Biol. 1964;7:1-16.

18. van Gestel J, Weissing FJ, Kuipers OP, Kovács ÁT. Density of founder cells affects spatial pattern formation and cooperation in Bacillus subtilis biofilms. ISME J. 2014;8:2069-79.

19. Nadell CD, Foster KR, Xavier JB. Emergence of spatial structure in cell groups and the evolution of cooperation. PLoS Comput Biol. 2010;6:e1000716.

20. Momeni B, Waite AJ, Shou W. Spatial self-organization favors heterotypic cooperation over cheating. Elife. 2013;2:e00960.

21. Dragoš A, Lakshmanan N, Martin M, Horváth B, Maróti G, García $\mathrm{CF}$, et al. Evolution of exploitative interactions during diversification in Bacillus subtilis biofilms. FEMS Microbiol Ecol. 2018;94:fix 155.

22. Ellis CN, Traverse CC, Mayo-Smith L, Buskirk SW, Cooper VS. Character displacement and the evolution of niche complementarity in a model biofilm community. Evolution. 2015;69:283-93. 
23. Poltak SR, Cooper VS. Ecological succession in long-term experimentally evolved biofilms produces synergistic communities. ISME J. 2011;5:369-78.

24. Goymer P, Kahn SG, Malone JG, Gehrig SM, Spiers AJ, Rainey PB. Adaptive divergence in experimental populations of Pseudomonas fluorescens. II. Role of the GGDEF regulator WspR in evolution and development of the wrinkly spreader phenotype. Genetics. 2006;173:515-26.

25. Rainey PB, Rainey K. Evolution of cooperation and conflict in experimental bacterial populations. Nature. 2003;425:72-4.

26. West SA, Griffin AS, Gardner A, Diggle SP. Social evolution theory for microorganisms. Nat Rev Microbiol. 2006;4:597-607.

27. Hardin G. The tragedy of the commons. Science. $1968 ; 162: 1243-8$.

28. Martin M, Hölscher T, Dragoš A, Cooper VS, Kovács ÁT. Laboratory evolution of microbial interactions in bacterial biofilms. J Bacteriol. 2016;198:2564-71.

29. Traverse CC, Mayo-Smith LM, Poltak SR, Cooper VS. Tangled bank of experimentally evolved Burkholderia biofilms reflects selection during chronic infections. Proc Natl Acad Sci USA. 2013;110:E250-9.

30. Madsen JS, Lin YC, Squyres GR, Price-Whelan A, Torio A, de S, et al. Facultative control of matrix production optimizes competitive fitness in Pseudomonas aeruginosa PA14 biofilm models. Appl Environ Microbiol. 2015;81:8414-26.

31. Rainey PB, Travisano M. Adaptive radiation in a heterogeneous environment. Nature. 1998;394:69-72.

32. Kreft JU. Biofilms promote altruism. Microbiology. 2004;150:2751-60.

33. Lyons NA, Kolter R. A single mutation in rapP induces cheating to prevent cheating in Bacillus subtilis by minimizing public good production. Commun Biol. 2018;1:133.

34. Veening JW, Igoshin OA, Eijlander RT, Nijland R, Hamoen LW, Kuipers OP. Transient heterogeneity in extracellular protease production by Bacillus subtilis. Mol Syst Biol. 2008;4:1-15.

35. Davidson FA, Seon-Yi C, Stanley-Wall NR. Selective heterogeneity in exoprotease production by Bacillus subtilis. PLoS One. 2012;7:e38574.

36. Kovács ÁT. Bacillus subtilis. Trends Microbiol. 2019;27:724-5.

37. Kovács ÁT, Dragoš A. Evolved biofilm: review on the experimental evolution studies of Bacillus subtilis pellicles. J Mol Biol. 2019;431:4749-59.

38. Hölscher T, Bartels B, Lin Y-C, Gallegos-Monterrosa R, PriceWhelan A, Kolter R, et al. Motility, chemotaxis and aerotaxis contribute to competitiveness during bacterial pellicle biofilm development. J Mol Biol. 2015;427:3695-708.
39. Dragoš A, Kiesewalter H, Martin M, Hsu C-Y, Hartmann R, Wechsler T, et al. Division of labor during biofilm matrix production. Curr Biol. 2018;28:1903-13.

40. Chai Y, Chu F, Kolter R, Losick R. Bistability and biofilm formation in Bacillus subtilis. Mol Microbiol. 2008;67:254-63.

41. Koo BM, Kritikos G, Farelli JD, Todor H, Tong K, Kimsey H, et al. Construction and analysis of two genome-scale deletion libraries for Bacillus subtilis. Cell Syst. 2017;4:291-305.e7.

42. Rueden CT, Schindelin J, Hiner MC, DeZonia BE, Walter AE, Arena ET, et al. ImageJ2: ImageJ for the next generation of scientific image data. BMC Bioinforma. 2017;18:529.

43. Zhu B, Stülke J. SubtiWiki in 2018: from genes and proteins to functional network annotation of the model organism Bacillus subtilis. Nucleic Acids Res. 2018;46:D743-8.

44. Dragoš A, Martin M, Garcia CF, Kricks L, Pausch P, Heimerl T, et al. Collapse of genetic division of labour and evolution of autonomy in pellicle biofilms. Nat Microbiol. 2018;3:1451-60.

45. Helmann JD. Bacillus subtilis extracytoplasmic function (ECF) sigma factors and defense of the cell envelope. Curr Opin Microbiol. 2016;30:122-32.

46. Kim W, Racimo F, Schluter J, Levy SB, Foster KR. Importance of positioning for microbial evolution. Proc Natl Acad Sci USA. 2014;111:E1639-47.

47. Cao M, Helmann JD. The Bacillus subtilis extracytoplasmicfunction $\sigma \mathrm{X}$ factor regulates modification of the cell envelope and resistance to cationic antimicrobial peptides. J Bacteriol. 2004;186:1136-46.

48. Murray EJ, Strauch Ma, Stanley-Wall NR. SigmaX is involved in controlling Bacillus subtilis biofilm architecture through the AbrB homologue Abh. J Bacteriol. 2009;191:6822-32.

49. Hentzer M, Teitzel GM, Balzer GJ, Heydorn A, Molin S, Givskov M, et al. Alginate overproduction affects Pseudomonas aeruginosa biofilm structure and function. $\mathrm{J}$ Bacteriol. 2001;183:5395-401.

50. Stilwell P, Lowe C, Buckling A. The effect of cheats on siderophore diversity in Pseudomonas aeruginosa. J Evol Biol. 2018;31:1330-9.

51. Kümmerli R, Brown SP. Molecular and regulatory properties of a public good shape the evolution of cooperation. Proc Natl Acad Sci USA. 2010;107:18921-6.

52. Niehus R, Picot A, Oliveira NM, Mitri S, Foster KR. The evolution of siderophore production as a competitive trait. Evolution. 2017;71:1443-55.

53. Dumas Z, Kümmerli R. Cost of cooperation rules selection for cheats in bacterial metapopulations. J Evol Biol. 2012;25:473-84. 